\title{
Remote plasma sputtering of indium tin oxide thin films for large area flexible electronics
}

\author{
A.D. Yeadon, S.J. Wakeham, H.L. Brown, M.J. Thwaites \\ M.J. Whiting, M.A. Baker
}

\section{A b s tra c t}

Indium tin oxide (ITO) thin films with a specific resistivity of $3.5 \times 10^{-4} \Omega \mathrm{cm}$ and average visible light transmission (VLT) of $90 \%$ have been reactively sputtered onto A4 Polyethylene terephthalate (PET), glass and silicon substrates using a remote plasma sputtering system.

This system offers independent control of the plasma density and the target power enabling the effect of the plasma on ITO properties to be studied. Characterization of ITO on glass and silicon has shown that increasing the plasma density gives rise to a decrease in the specific resistivity and an increase in the optical band gap of the ITO films. Samples deposited at plasma powers of $1.5 \mathrm{~kW}$, $2.0 \mathrm{~kW}$ and $2.5 \mathrm{~kW}$ and optimized oxygen flow rates exhibited specific resistivity values of $3.8 \times 10^{-4} \Omega \mathrm{cm}, 3.7 \times 10^{-4} \Omega \mathrm{cm}$ and $3.5 \times 10^{-4} \Omega \mathrm{cm}$ and optical gaps of $3.48 \mathrm{eV}, 3.51 \mathrm{eV}$ and $3.78 \mathrm{eV}$ respectively.

The increase in plasma density also influenced the crystalline texture and the VLT increased from 70 to $95 \%$, indicating that more oxygen is being incorporated into the growing film. It has been shown that the remote plasma sputter technique can be used in an in-line process to produce uniform ITO coatings on PET with specific resistivities of between $3.5 \times 10^{-4}$ and $4.5 \times 10^{-4} \Omega \mathrm{cm}$ and optical transmission of greater than $85 \%$ over substrate widths of up to $30 \mathrm{~cm}$.

\section{Introduction}

Indium tin oxide (ITO) is a commonly used transparent conducting oxide (TCO) with applications as a transparent electrode for flat panel displays [1], photovoltaic devices [2] and as an infrared mirror in energy saving windows [3]. ITO offers the best combination of electrical conductivity and visible light transmission with excellent environmental stability, reproducibility and surface morphology $[1,3]$.

ITO is based on indium oxide (In2O3), a wide optical gap ( $\approx 3.6 \mathrm{eV}[4]$ ) semiconductor material which, as a result of being $n$-type degenerate, is electrically conducting. Two explanations given in the literature for the source of the free carriers in indium oxide are (a) oxygen vacancies [5] and (b) a hydrogen doping mechanism [6-8]. In ITO, the free charge carrier (electron) concentration is further increased by substitutionally doping $\mathrm{In}^{3+}$ sites with $\mathrm{Sn}^{4+}[9]$. The doping efficiency is related to the energy delivered to the growing film during deposition. To effectively dope the ITO, commercial films are deposited at high temperature $[10,11]$. 
The recent interest in flexible electronic devices has increased demand for processes offering low temperature deposition of high quality ITO films onto large area polymeric substrates [12]. The move to polymeric substrates is driven by their relatively low cost, mechanical flexibility, and light weight [2]. These properties not only make flexible devices attractive to the end customer, but also enable efficient mass manufacturing using 'roll to roll' processes [10]. However, due to the thermal sensitivity of polymers, sputter deposition of high quality ITO remains an area of intense research [11].

To meet the requirements of flexible displays, research has focused on methods of depositing materials at low temperature, ideally less than $78^{\circ} \mathrm{C}$ (the glass transition temperature of PET) [10]. Wakeham et al. [11] deposited ITO with a specific resistivity of $3.8 \times 10^{-4} \Omega \mathrm{cm}$ and average visible (400-750 $\mathrm{nm}$ ) light transmission (VLT) greater than $90 \%$, on to a range of flexible substrates ( $<15 \mathrm{~mm}$ diameter bend radius) using a remote plasma system. The high quality of the ITO was attributed to the increased energy at the substrate surface as a result of the high density plasma $\left(10^{13}\right.$ ions $\left.\mathrm{cm}^{-3}\right)$, used in this sputtering process.

The remote plasma system benefits from independent plasma generation and target biasing, resulting in control of the flux of ions and their energies. The ability to generate a high flux of low energy ions has been shown to be beneficial for most thin film deposition processes [13]. This investigation extends the original work of Wakeham et al. [11] with the aim of developing a large area ITO process for use on polymeric substrates. After initial optimization of the deposition parameters, two sets of ITO films were deposited onto glass and silicon to explore the effects of varying the plasma power for films deposited under optimized and oxygen depleted conditions. Firstly, at a depleted oxygen flow rate of $18 \mathrm{sccm}$ a set of films was deposited using plasma powers in the range of 2.0-3.5 kW. Then a second set of films was deposited at the optimum oxygen flow rate of $25 \mathrm{sccm}$ employing plasma powers in the range of 1.5-2.5 kW. For comparison, a sample radiatively heated to $200{ }^{\circ} \mathrm{C}$ was deposited at this same optimum oxygen flow rate. Finally, the optimized process was used to deposit ITO onto A4 PET substrates to demonstrate the compatibility of the process with polymeric substrates.

\section{Experimental Technique}

\section{Thin film deposition}

ITO films were reactively sputtered from a metallic 90:10 wt.\% In:Sn (purity 99.99\%) target at different oxygen flow rates and plasma powers using an in-line large area remote plasma system (details of the technology are discussed elsewhere $[11,14,15]$ ) onto glass and silicon substrates. A schematic diagram of the system is shown in Fig. 1. 


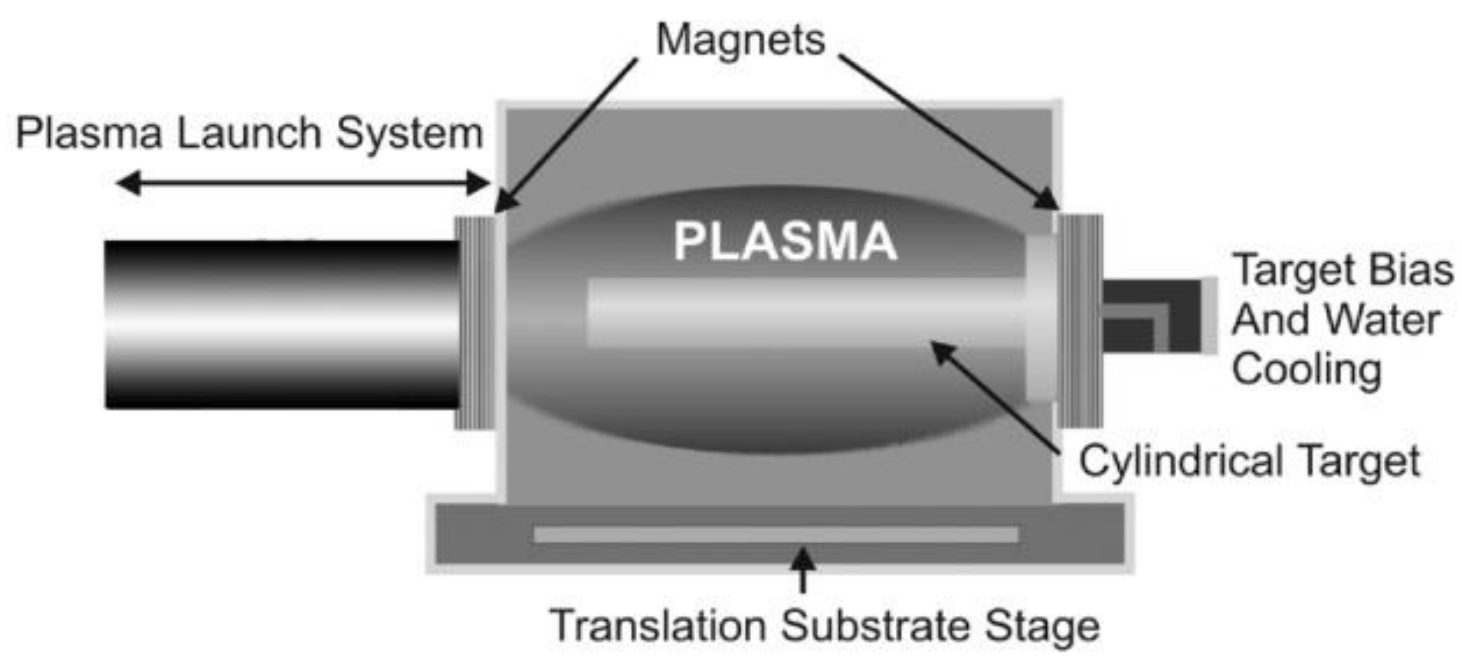

Fig. 1. A schematic diagram of the remote plasma system fitted with a $50 \mathrm{~cm}$ cylindrical target. The plasma is generated in the plasma launch system (PLS) and a magnetic field is used to form a plasma envelope around the target. In this diagram, the substrate travels in plane.

The remote plasma sputtering process uses a magnetically confined, remotely generated argon plasma to provide a uniform distribution of ions at the target surface. The process is resistant to target poisoning and is very stable as a result. The in-line system is fitted with a load lock and translating substrate holder (positioned $30 \mathrm{~cm}$ below the target) enabling a high sample throughput.

Prior to deposition the substrates were cleaned using an EcoClear ${ }^{\circledast}$ cleaning solution and dried with dry nitrogen gas. The chamber was evacuated to a base pressure of $6 \times 10^{-4} \mathrm{~Pa}$ before being filled with argon to a process pressure of $0.3 \mathrm{~Pa}$. The substrates were treated with a low power plasma (no DC bias was applied to the target/substrate) to desorb volatile surface contaminants such as hydrocarbon species and adsorbed water. The target was prepared for the coating process by presputtering in $\mathrm{Ar}$ and then in the $\mathrm{Ar}+\mathrm{O}_{2}$ gas mixture required for the subsequent coating stage of the process. 
Table 1. The deposition parameters for the two sets of ITO coatings deposited at oxygen flow rates of 25 sccm (sample numbers 25-1 to 25-4) and 18 sccm (sample numbers 18-1 to 18-4).

\begin{tabular}{|l|l|l|l|l|l|l|l|l|}
\hline Sample & $\mathbf{2 5 - 1}$ & $\mathbf{2 5 - 2}$ & $\mathbf{2 5 - 3}$ & $\mathbf{2 5 - 4}$ & $\mathbf{1 8 - 1}$ & $\mathbf{1 8 - 2}$ & $\mathbf{1 8 - 3}$ & $\mathbf{1 8 - 4}$ \\
\hline $\begin{array}{l}\text { Argon } \\
(\mathbf{s c c m})\end{array}$ & 70 & 70 & 70 & 70 & 70 & 70 & 70 & 70 \\
\hline $\begin{array}{l}\text { RF plasma } \\
\text { power (kW) }\end{array}$ & 1.5 & 2.0 & 2.5 & 2.0 & 2.0 & 2.5 & 3.0 & 3.5 \\
\hline $\begin{array}{l}\text { Deposition } \\
\text { temperature } \\
\left({ }^{\circ} \mathbf{C}\right)\end{array}$ & ambient & ambient & ambient & 200 & ambient & ambient & ambient & ambient \\
\hline $\begin{array}{l}\text { Ion current } \\
(\mathbf{A})\end{array}$ & 3.4 & 4.4 & 5.4 & 4.2 & 4.2 & 5.3 & 6.1 & 6.9 \\
\hline DC bias (V) & 294 & 230 & 185 & 240 & 240 & 189 & 160 & 147 \\
\hline $\begin{array}{l}\text { Oxygen } \\
(\mathbf{s c c m})\end{array}$ & 25 & 25 & 25 & 25 & 18 & 18 & 18 & 18 \\
\hline $\begin{array}{l}\text { Thickness } \\
(\mathbf{n m})\end{array}$ & 350 & 380 & 450 & 320 & 400 & 400 & 400 & 400 \\
\hline
\end{tabular}

After the initial process optimization (described in Section 3.1), two sample sets were used to investigate the effects of the plasma power at different oxygen flow rates. The process parameters used to deposit these samples are given in Table 1. The samples have been numbered using a system incorporating the $\mathrm{O}_{2}$ flow rate as an identifier for the two different sample sets (i.e. samples 18-1 to 18-4 for the samples deposited at $18 \mathrm{sccm}$ and 25-1 to 25-4 for the samples deposited at $25 \mathrm{sccm}$ $\mathrm{O}_{2}$ ). All samples were deposited onto unheated substrates except for sample 25-4 which was radiatively heated to $200{ }^{\circ} \mathrm{C}$ during thin film deposition. The samples were not annealed after deposition.

\section{Characterization}

Glass and silicon were initially employed as substrates for characterization purposes. ITO films deposited onto glass were examined using an AvaSpec UV-Vis-NIR spectrophotometer (spectral range 300-1100 nm) and a Jandel Engineering Ltd four point probe for their optical transmittance and specific resistivity respectively. The film thickness was measured using a Taylor Hobson Talystep profilometer.

Structural characterization was performed on ITO films deposited onto silicon using a Panalytical Xpert Pro X-ray Diffractometer using a Cu Ka (1.5418 ̊̊) source running at a voltage of $40 \mathrm{kV}$ and current of $30 \mathrm{~mA}$. The $2 \theta$ range scanned was $10-65^{\circ}$ with a $2 \theta$ step size of $0.017^{\circ}$. The average grain sizes were determined from the (222) peak employing the De Keijser single line method [16,17], which involves a least squares fitting of the XRD peaks using a pseudo-Voigt function and then applying the Scherrer equation [21] to the Lorentzian component of the peak.

To perform carrier concentration and mobility measurements, the films were etched into a cloverleaf shape and analyzed using the Van Der Pauw method on an Accent HL5500 Hall system [18]. 


\section{Results}

\subsection{Initial process setup - influence of oxygen on optical and electrical properties}

Before studying the effects of plasma launch power on the ITO properties, samples were deposited at oxygen flow rates from 0 to $25 \mathrm{sccm}$ with increments of $5 \mathrm{sccm}$ between depositions. The specific resistivity and optical transmission were measured to allow the optimum oxygen flow rate to be determined. A constant $2.0 \mathrm{~kW}$ plasma launch power and $1.0 \mathrm{~kW}$ target power were used throughout this initial optimization process.

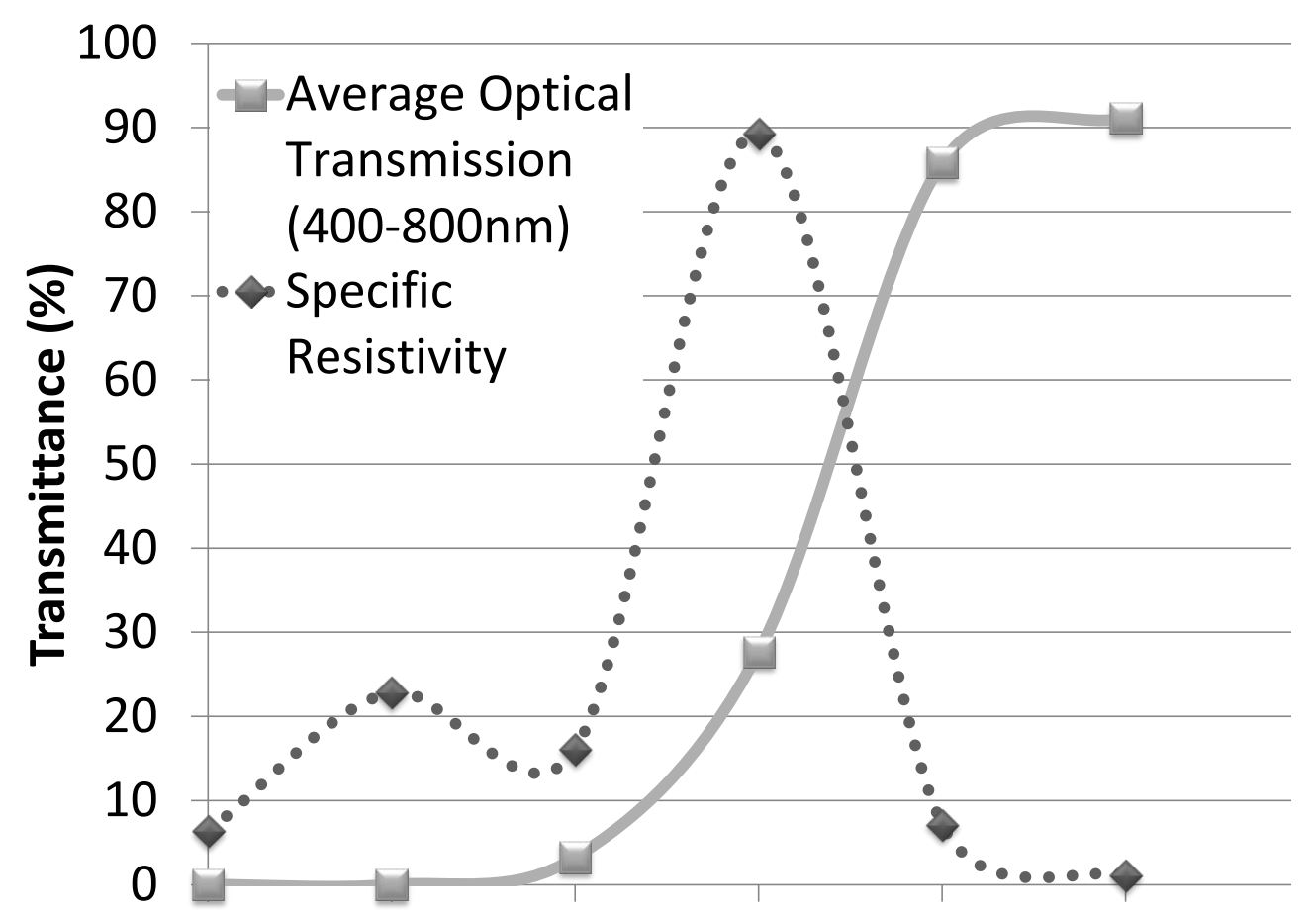

Fig. 2. A plot showing the specific resistivity and VLT of the ITO films, as a function of oxygen flow rate. Beyond 25 sccm the specific resistivity is expected to increase, however at a plasma power of $2.0 \mathrm{~kW}$, the system became unstable for oxygen flow rates greater than $25 \mathrm{sccm}$, preventing the collection of further data.

Fig. 2 shows that with increasing $\mathrm{O}_{2}$ flow rate, the ITO approaches full stoichiometry, resulting in an increase in visible light transmission (VLT) and 2 orders of magnitude decrease in specific resistivity between 15 and $25 \mathrm{sccm}$. At $25 \mathrm{sccm}$ the ITO coating exhibits VLT of $92 \%$ and specific resistivity of $3.8 \times 10^{-4} \Omega \mathrm{cm}$. These results show that the process is stable and capable of producing high quality ITO.

\subsection{Characterization of IT0 samples deposited at flow rates of 18 and 25} $\operatorname{sccm}$

All the ITO films deposited had thicknesses in the range 320-450 nm (Table 1). The effects of plasma power were investigated on films deposited under oxygen depleted conditions (oxygen flow rate of $18 \mathrm{sccm}$ ). The optical transmission data for ITO deposited at plasma powers from 2.0 to $3.5 \mathrm{~kW}$ (Fig. 3) shows that raising the plasma launch power results in an increase in the optical transmission. From Fig. 2, it can be seen that a similar effect is observed as the oxygen flow rate is increased. 


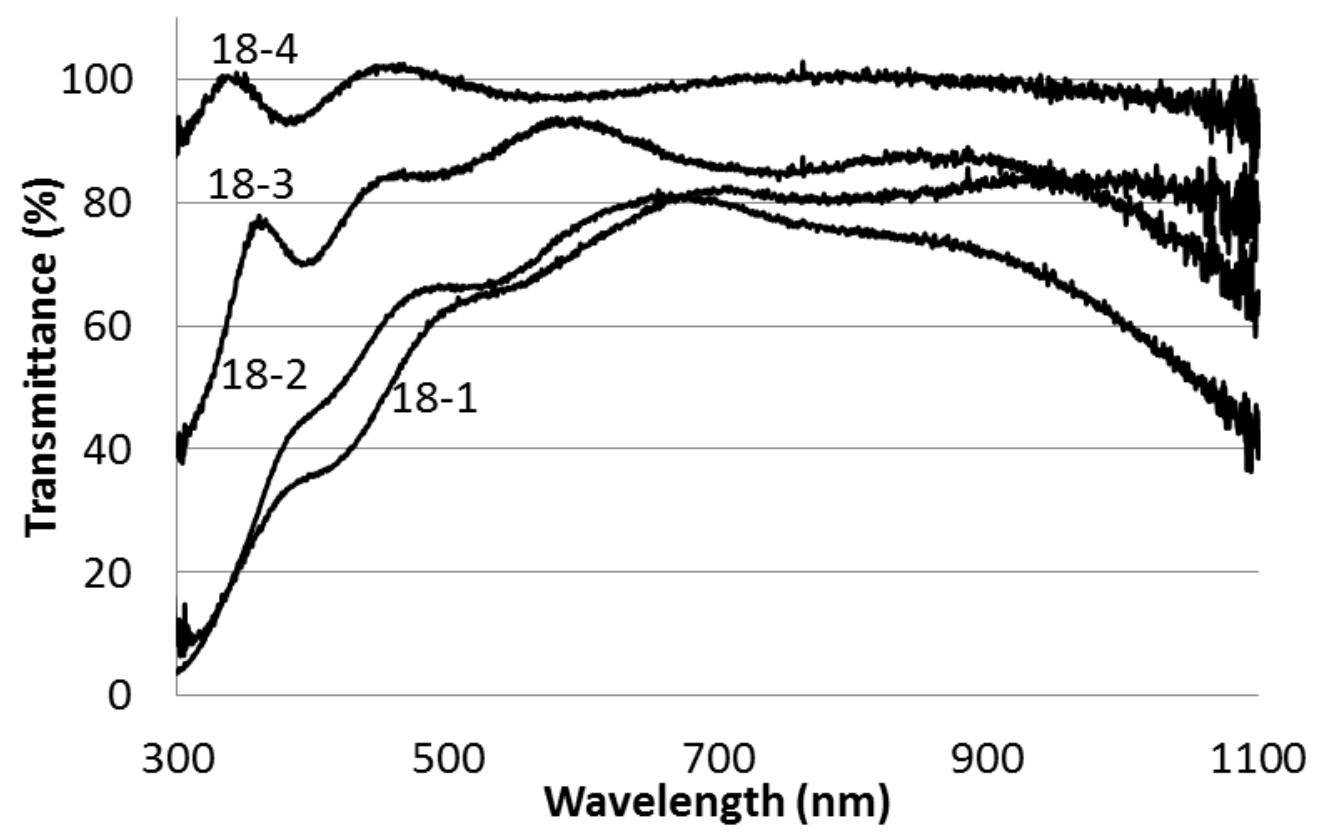

Fig. 3. Optical transmission spectra for the ITO films deposited at an oxygen flow of $18 \mathrm{sccm}$ with plasma launch powers ranging from 2.0 to $3.5 \mathrm{~kW}$, referenced to the glass substrates. The transmission is seen to improve as the plasma launch power is increased.

Fig. 4 shows the optical transmission data for ITO deposited at the optimal oxygen flow rate of $25 \mathrm{sccm}$ for plasma powers from 1.5 to $2.5 \mathrm{~kW}$. For comparison, the result for the sample deposited at the same oxygen flow rate and plasma power of $2.0 \mathrm{~kW}$, but heated to $200{ }^{\circ} \mathrm{C}$ during film growth (sample 25-4) is also presented. It can be seen that ITO samples deposited at $1.5 \mathrm{~kW}$ and $2.0 \mathrm{~kW}$ have almost identical transmission spectra. However, as the plasma launch power is increased to $2.5 \mathrm{~kW}$, the absorption edge of the ITO moves to shorter wavelengths, consistent with an increase in the optical band gap. The optical band gap increases further for the sample deposited at elevated temperature. 


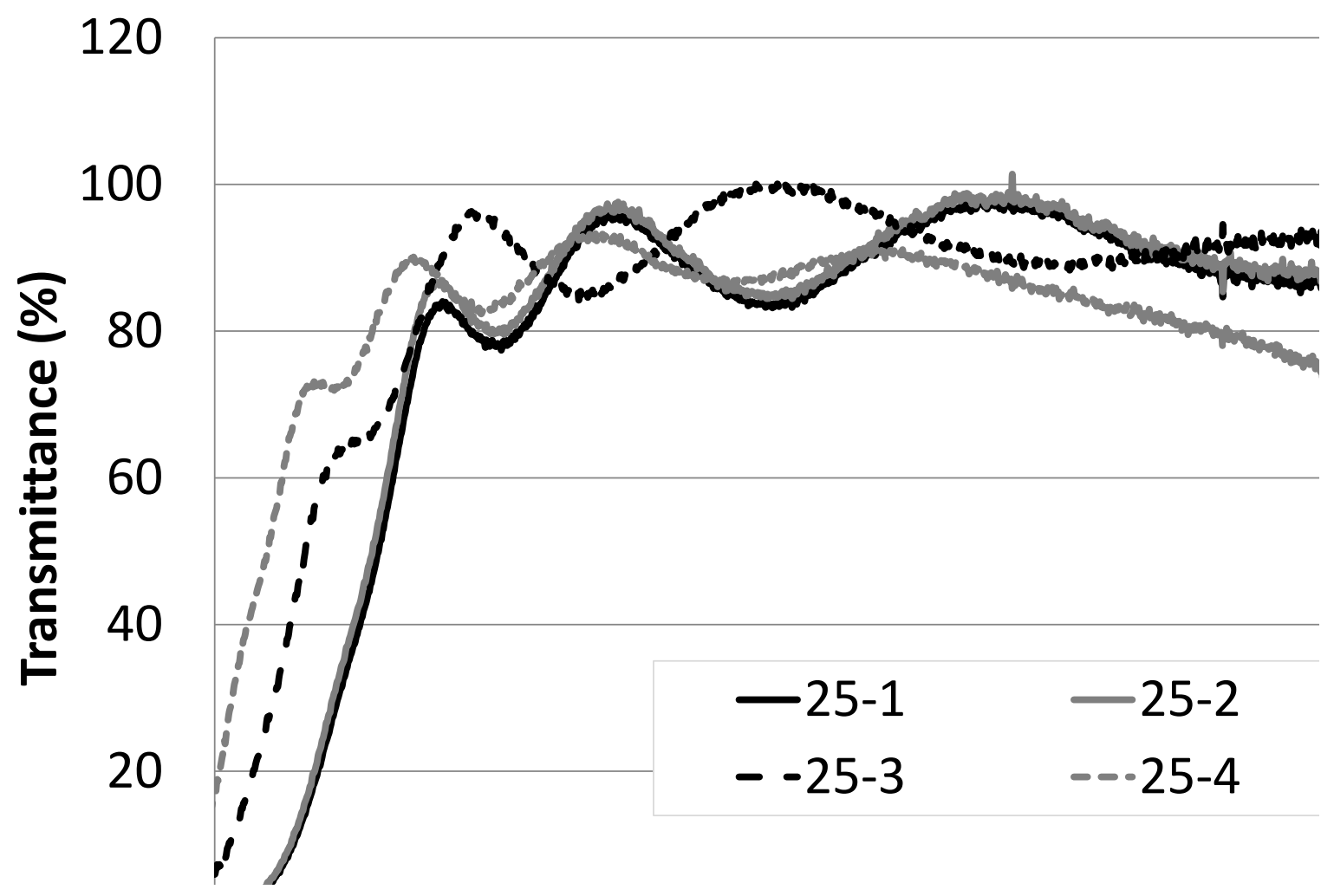

Fig. 4. Optical transmission spectra for the ITO films deposited at an oxygen flow of $25 \mathrm{sccm}$ with plasma powers ranging from 1.5 to $2.5 \mathrm{~kW}$, referenced to the glass substrates. The transmission of the heated sample (25-4) shows the spectrum of ITO with a high carrier concentration.

The Tauc relationship (Eq. (1)) was used to calculate the ITO optical band gap, Eg.

$$
(\alpha h u)^{2}=C\left(h u-E_{g}\right)
$$

Where $\alpha$ is the absorption coefficient, $\mathrm{h}$ is Plank's constant, $\mathrm{v}$ is the frequency and $\mathrm{C}$ is a constant $[19,20]$. The optical gap for the $25 \mathrm{sccm}$ oxygen samples is shown to increase from $3.48 \mathrm{eV}$ to $3.91 \mathrm{eV}$ with increased plasma launch power and heating (Fig. 5). This is consistent with the Burstein-Moss effect which predicts a widening of the optical band gap as more low energy states in the conduction band are filled by free carriers [21,22]. 


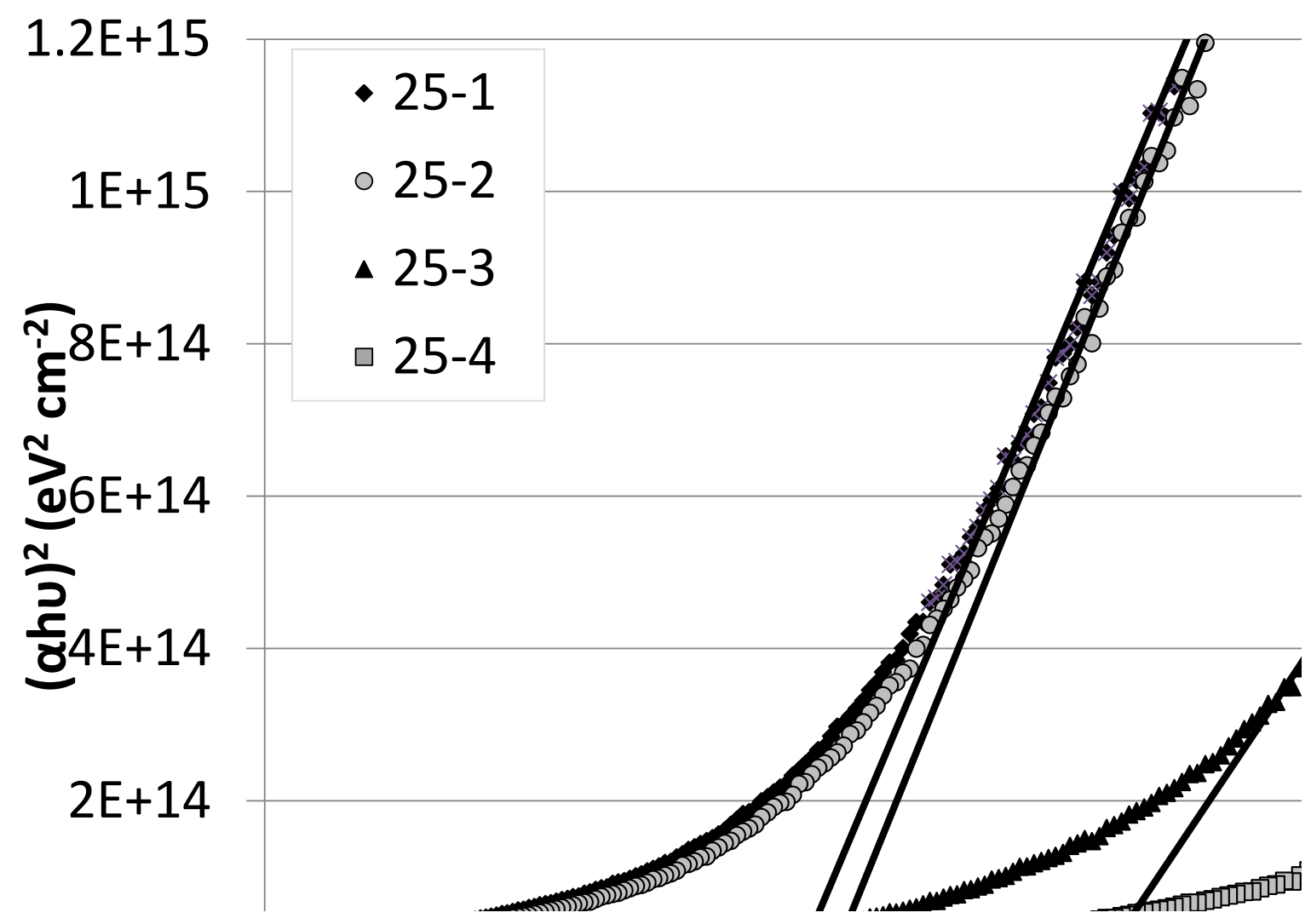

Fig. 5. Plots of ( $\alpha$ hu)2 as a function of hu for the ITO films deposited at an oxygen flow of $25 \mathrm{sccm}$. The optical band gap is predicted by extrapolating the gradient from the linear part of the plot to the energy axis. The widening of the band gap is indicative of an increase in carrier concentration.

With regard to electrical properties, both sets of samples show a decrease in specific resistivity with an increase in plasma power. The specific resistivity of samples 18-2, 18-3 and 18-4 was found to be $12.6 \times 10^{-4} \Omega \mathrm{cm}, 11.6 \times 10^{-4} \Omega \mathrm{cm}$ and $9.2 \times 10^{-4} \Omega \mathrm{cm}$ respectively. A more extensive set of electrical properties data, including Hall measurements, was recorded for the samples deposited at the oxygen flow rate of $25 \mathrm{sccm}$ and the results are presented in Table 2.

Table 2. A table showing the electrical properties of the ITO coatings deposited at an oxygen flow rate of $25 \mathrm{sccm}$ on glass substrates.

\begin{tabular}{|c|c|c|c|c|}
\hline Samples & $25-1$ & $25-2$ & $25-3$ & $25-4$ \\
\hline Resistivity $\times 10^{-4}(\mathrm{ohm} \mathrm{cm})$ four point probe & 3.8 & 3.6 & 3.5 & 1.9 \\
\hline Resistivity $\times 10^{-4}(\mathrm{ohm} \mathrm{cm})$ Hall measurement & 3.8 & 3.7 & 3.5 & 2.6 \\
\hline Mobility $\mathrm{cm}^{2} / \mathrm{V}$-s & 50 & 45 & 43 & 42 \\
\hline Carrier concentration $\times 10^{20} / \mathrm{cm}^{3}$ & 3.5 & 3.7 & 4.2 & 5.6 \\
\hline
\end{tabular}


The Hall measurements indicate that the higher conductivity occurs due to an increase in the carrier concentration, consistent with the Burstein-Moss effect, as shown by the band gap widening in Fig. 5. This result agrees with the literature where the main increase in ITO conductivity is attributed to an increase in free carriers as a result of the effective substitutional doping of $\ln ^{3+}$ sites with $\mathrm{Sn}^{4+}$ [9].

X-ray diffraction has been used to investigate the effects of increasing the plasma launch power and heating on the crystal structure of the material. As the doping of ITO is strongly influenced by crystallinity, a strong correlation between the grain size and the specific resistivity of the material would be expected.

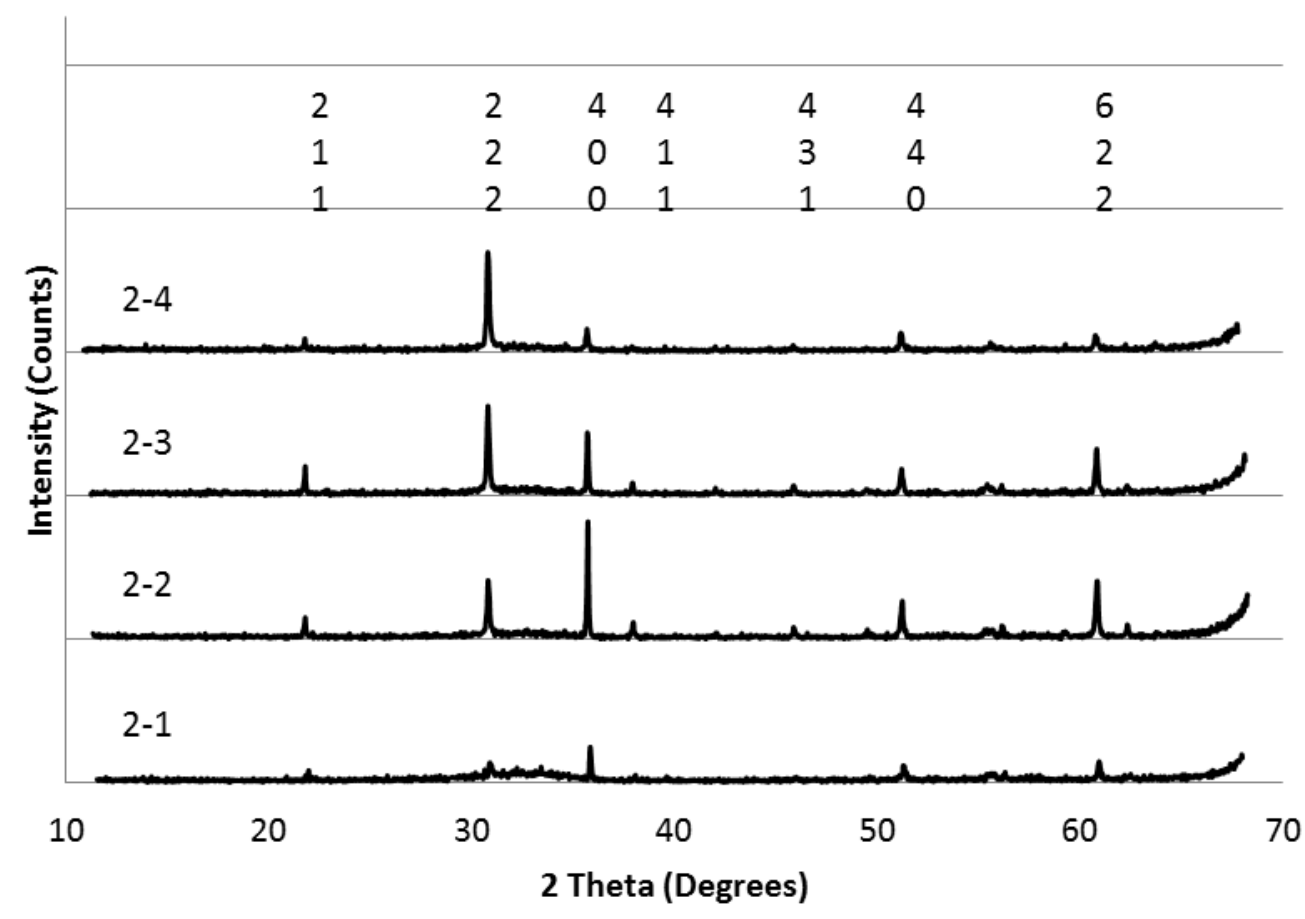

Fig. 6. XRD diffractograms showing the effect of plasma power on the structure of ITO films deposited onto silicon substrates at an oxygen flow rate of $18 \mathrm{sccm}$. The structure changes from a (400) to (222) texture with increasing plasma power.

For the samples deposited at the depleted oxygen concentration $(18 \mathrm{sccm})$, the XRD diffractograms given in Fig. 6 show that at plasma powers between 2.5 and $3.5 \mathrm{~kW}$, the samples have a structure which is nanocrystalline. However, the additional presence of an amorphous 'hump' in the diffractogram for sample 18-1, deposited at $2.0 \mathrm{~kW}$, indicates that this ITO film has a mixed amorphous/nanocrystalline structure. All samples exhibited average grain sizes in the range of 55-65 $\mathrm{nm}$ (on both silicon and glass substrates) and it can be seen that as the plasma density is increased, the texture changes from (400) to (222).

The XRD diffractograms for the (unheated) samples deposited at optimum oxygen concentration $(25 \mathrm{sccm})$ show a similar behaviour to those deposited at lower oxygen concentrations. At low plasma powers, of $1.5 \mathrm{~kW}$ and $2.0 \mathrm{~kW}$, the ITO films exhibit a mixed amorphous/nanocrystalline structure and at the higher power of $2.5 \mathrm{~kW}$ the structure changes to purely nanocrystalline. At a power of $2.0 \mathrm{~kW}$, heating the ITO film to $200^{\circ} \mathrm{C}$ during deposition leads to a purely nanocrystalline 
structure being formed. Both of the nanocrystalline coatings had an average grain size of $35-40 \mathrm{~nm}$ (on both glass and silicon substrates). With regard to texture, at the highest power of $2.5 \mathrm{~kW}$, a (222) texture is observed, while the sample deposited at $2.0 \mathrm{~kW}$ and heated to $200^{\circ} \mathrm{C}$, shows a (400) texture.

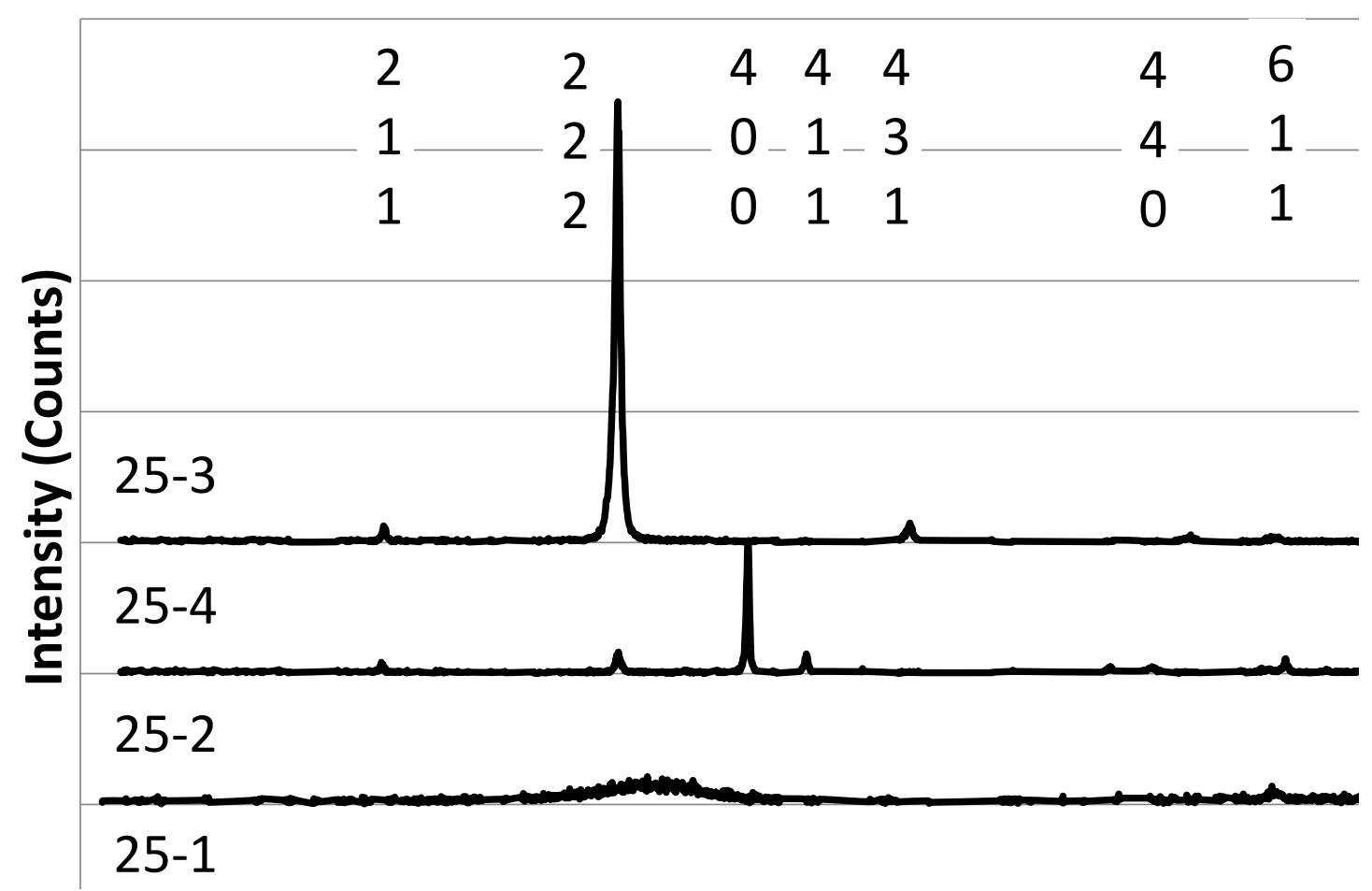

Fig. 7. XRD diffractograms showing the effect of plasma power on the structure of ITO films deposited onto silicon substrates at an oxygen flow rate of $25 \mathrm{sccm}$.

Comparing the nanostructures formed at 18 and $25 \mathrm{sccm}$ oxygen, it can be seen that a similar (222) textured nanocrystalline structure is formed through depositing at a high plasma power at lower oxygen flow rates or a medium plasma power at a higher oxygen concentration (Fig. 7).

\section{Deposition of IT0 on A4 PET}

Having developed an optimized process for deposition of ITO onto glass and silicon, a $100 \mathrm{~nm}$ thick ITO film was deposited onto an A4 PET sheet at a plasma power of $2.0 \mathrm{~kW}$ and an oxygen flow rate of $25 \mathrm{sccm}$ to demonstrate the compatibility of the process with organic substrates. 


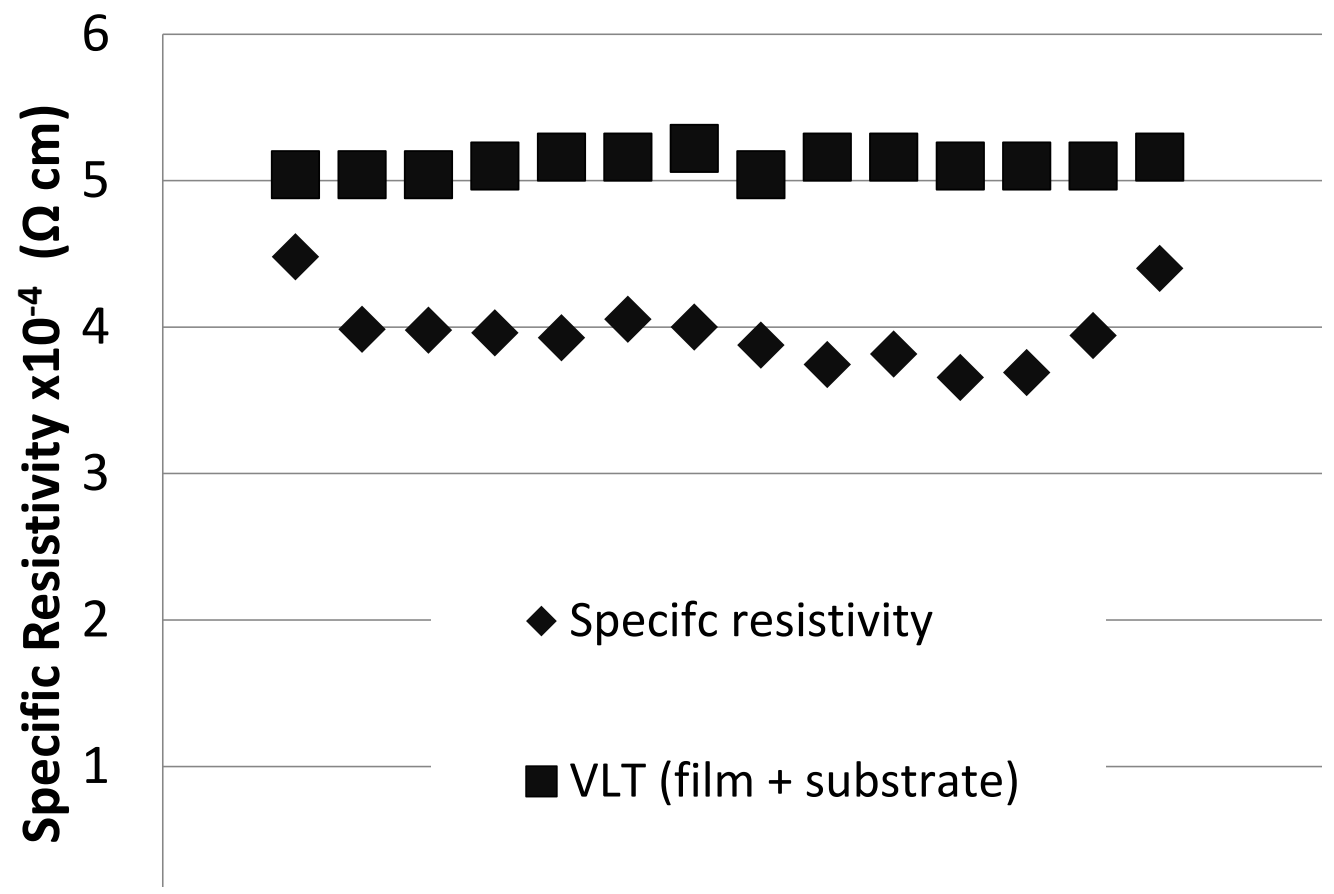

Fig. 8. A plot showing the specific resistivity and VLT of the ITO as a function of distance from the plasma source. The plot shows that the ITO is deposited uniformly along the line of the target, which is the most non-uniform direction in a web coating system.

Fig. 8 shows that the average specific resistivity of the deposited ITO was $3.96 \times 10^{-4} \Omega \mathrm{cm}$ with a variation of less than $\pm 10 \%$ over $26 \mathrm{~cm}$. Hence, a highly uniform deposition process is possible for a web coating system with well-designed apertures and gas distribution. The VLT was observed to be greater than $85 \%$ at all points on the film (Fig. 8). The sheet resistance of these ITO films was found to be $40 \Omega / \square$. With no active cooling of the steel backplate, $100 \mathrm{~nm}$ thick ITO films can easily be deposited onto PET sheets without degradation of the substrate. Hence, the deposition temperature is assumed to be less than $78^{\circ} \mathrm{C}$.

\section{Discussion}

This work has shown that increasing the plasma power at low oxygen flow rates results in a change of texture from (400) to (222) and an improvement in the ITO properties: i.e. a larger VLT and a reduction in specific resistivity. Such properties have all been seen to occur either as a result of increasing the oxygen flow rate, and hence an increased oxygen incorporation into the growing film, as seen from our results and those of other workers [23] or through depositing films using reactive oxygen species, such as ozone [24]. This latter result indicates that an increase in the oxygen incorporation could be achieved by an increase in the reactivity of the oxygen.

Our results have demonstrated that the high ion density $\left(10^{13}\right.$ ions $\left.\mathrm{cm} 3\right)$ in the remote plasma system is very effective at ionizing argon gas. Given that the first ionization energy of both indium and oxygen is lower than that of argon [25], the plasma is likely to generate indium and oxygen ions as well as reactive species such as ozone. Consequently, the improved ITO properties observed for increased plasma powers at low oxygen flow rates can be attributed to the increase in reactivity of the oxygen and indium as a result of the more energetic plasma conditions. The ability to increase 
the probability of the indium-oxygen reaction is significant for process stability as a lower oxygen flow rate would decrease the chance of process runaway occurring.

The depositions at optimum oxygen flow rates show a transition from amorphous/nanocrystalline to crystalline as the plasma power isincreased. The nanocrystalline films deposited at $2.5 \mathrm{~kW}$ exhibit an increased optical band gap, conductivity and carrier concentration compared to films deposited at lower plasma powers. This change in properties occurs as the $\mathrm{Sn}^{4+}$ becomes more active as an electron donor in the crystalline lattice as it subsitutionally dopes indium sites. The mobility of charge carriers in the ITO appears to decrease as the carrier concentration increases (Table 2). This is explained by an increased scattering from ionized impurities $\left(\mathrm{Sn}^{4+}\right.$ dopants) [26].

The formation of each of the textures observed for films deposited under different conditions can be explained by considering surface mobility and ion bombardment. The texture of a material is determined by the relative growth rate for each of the crystallographic planes present at the surface [27]. Under normal conditions with high surface mobility, ITO will grow with a structure which is predominantly (222) oriented [28]. From these experiments, it was found that for high surface mobility conditions (heating the sample), a (400) texture develops. The usual explanation of resputtering [29] seems unlikely, since any increase in plasma density results in an increase in the target current for the remote plasma system. As the films were deposited at constant target power, the bias on the target is reduced to compensate for the increased current, resulting in a higher flux of lower energy ions. The energies of the ions approach those of 'plasma only' conditions, which are too low to cause re-sputtering $[11,14,15]$. Petrov et al. [13] have shown that for TiN, a change in the ratio of the flux of ionized/atomic nitrogen can affect the texture of the thin films formed. They attributed this to the different diffusion coefficients of ions and atoms on the (100) and (111) surfaces. Their results are similar to the texture changes observed for our ITO films. Hence, it seems probable that at higher plasma powers, the increased flux of ions is influential in changing the ITO texture.

\section{Conclusion}

A remote plasma sputtering system which allows independent control of the plasma density and the target power has been employed to grow ITO thin films under different process conditions. In particular, the effect of plasma density on film properties and microstructure has been studied. Increasing the plasma density leads to a decrease in the specific resistivity and increase in the optical band gap of the ITO films. Samples deposited at plasma powers of $1.5 \mathrm{~kW}, 2.0 \mathrm{~kW}$ and $2.5 \mathrm{~kW}$ and optimized oxygen flow rates exhibited specific resistivity values of $3.8 \times 10^{-}{ }^{4} \Omega \mathrm{cm}, 3.7 \times 10^{-4} \Omega \mathrm{cm}$ and $3.5 \times 10^{-4} \Omega \mathrm{cm}$ and optical band gaps of $3.48 \mathrm{eV}, 3.51 \mathrm{eV}$ and $3.78 \mathrm{eV}$ respectively.

By growing ITO films at depleted oxygen concentrations, it has been shown that increasing the plasma power provides not only an energy source for increased adatom surface mobility and film densification, but also acts to increase the probability of chemical reactions through higher ionization of the reacting oxygen and indium species, leading to improved properties of the deposited ITO films. 
ITO films deposited onto A4 PET sheets exhibited a sheet resistance of $40 \Omega / \square$ and VLT of greater than $85 \%$. The variation of the specific resistivity measured across an A4 ITO coated sheet is better than $\pm 10 \%$ indicating that a highly uniform large area deposition process should be possible with further development.

\section{Acknowledgements}

The authors would like to acknowledge the EngD studentship fromPlasma Quest Ltd, the University of Surrey, and the EPSRC. Thanks must also go to Dr Peter Hockley for detailed discussions on the nature of the HiTUS system and all other members of the Plasma Quest team for their support.

\section{References}

[1] U. Betz, M. Kharrazi Olsson, J. Marthy, M.F. Escolá, F. Atamny, Surf. Coat. Tech. 200(2006) 5751.

[2] K.-H. Choi, J.-A. Jeong, J.-W. Kang, D.-G. Kim, J.K. Kim, S.-I. Na, D.-Y. Kim, S.-S. Kim,H.-K. Kim, Sol. Energy Mater. Sol. Cells 93 (2009) 1248.

[3] B.G. Lewis, D.C. Paine, MRS Bull. (2000) 23.

[4] A. Walsh, J.L.F. Da Silva, S.-H. Wei, C. Körber, A. Klein, L.F.J. Piper, A. DeMasi, K.E. Smith, G. Panaccione, P. Torelli, D.J. Payne, A. Bourlange, R.G. Egdell, Phys. Rev.Lett. 100 (2008) 167402.

[5] S. Li, X. Qiao, J. Chen, Mater. Chem. Phys. 98 (2006) 144.

[6] P.D.C. King, R.L. Lichti, Y.G. Celebi, J.M. Gil, R.C. Vilão, H.V. Alberto, J.P. Duarte, D.J.Payne, R.G. Egdell, I. McKenzie, Phys. Rev. B 80 (2009) 081201.

[7] A. Janotti, C.G. Van de Walle, Nat. Mater. 6 (2006) 44.

[8] M. Ando, M. Takabatake, E. Nishimura, F. Leblanc, K.- Onisawa, T. Minemura, J.Non-Cryst. Solids 198-200 (1996) 28.

[9] C.G. Granqvist, A. Hultåker, Thin Solid Films 411 (2002) 1.

[10] M.-C. Choi, Y. Kim, C.-S. Ha, Prog. Polym. Sci. 33 (2008) 581.

[11] S.J. Wakeham, M.J. Thwaites, B.W. Holton, C. Tsakonas, W.M. Cranton, D.C.Koutsogeorgis, R. Ranson, Thin Solid Films 518 (2009) 1355.

[12] Y.Z. You, Y.S. Kim, D.H. Choi, H.S. Jang, J.H. Lee, D. Kim, Mater. Chem. Phys. 107(2008) 444.

[13] I. Petrov, P.B. Barna, L. Hultman, J.E. Greene, J. Vac. Sci. Technol., A 21 (2003) S117.

[14 ] P. Hockley, M.J. Thwaites, UK Patent No. GB2419894, 2004. 
[15] M.J. Thwaites, UK Patent No. GB2343992, 1998.

[16] T.H. De Keijser, E.J. Mittemeijer, H.C.F. Rozendaal, J. Appl. Crystallogr. 16 (1983)309.

[17] P. Scherrer, N.G.W. Gottingen, Math-Phys. KI. 2 (1918) 96.

[18] T.I. Kamins, J. Appl. Phys. 42 (1971) 4357.

[19] J. Tauc, R. Grigorovici, A. Vancu, Phys. Status Solidi B 15 (1966) 627.

[20] Z.Z. You, Mater. Lett. 61 (2007) 3809.

[21] A. Sarkar, S. Ghosh, S. Chaudhuri, A.K. Pal, Thin Solid Films 204 (1991) 255.

[22] A. Walsh, J.L.F. Da Silva, S.-H. Wei, Phys. Rev. B 78 (2008) 075211.

[23] H.-C. Lee, O. Ok Park, Vacuum 77 (2004) 69.

[24] A.H.M.Z. Alam, K. Sasaki, T. Hata, Thin Solid Films 281-282 (1996) 209.

[25] D.R. Lide, CRC Handbook of Chemistry and Physics (Internet Version 2011), CRC Press/Taylor and Francis, Boca Raton, FL, 2011.

[26] H.-C. Lee, O. Ok Park, Vacuum 75 (2004) 275.

[27] A. Van der Drift, Philips Res. Rep 22 (1967) 267.

[28] N. Nadaud, N. Lequeux, M. Nanot, J. Jové, T. Roisnel, J. Solid State Chem. 135 (1998) 140.

[29] M. Kamei, Y. Shigesato, S. Takaki, Thin Solid Films 259 (1995) 38. 